Journal of ISMAC (2020)

Vol.02/ No. 02

Pages: $118-128$

http://irojournals.com/iroismac/

DOI: https://doi.org/10.36548/jismac.2020.2.005

\title{
Novel Algorithm for Service Composition and QOS Enhancements in Composite Computing Services
}

\author{
Dr. A. Pasumpon Pandian, \\ Professor, \\ Computer Science Engineering, \\ KGiSL Institute of Technology, \\ Coimbatore, India. \\ Email id: pasumponpandian32@gmail.com
}

\begin{abstract}
The swift progress in the computing set up by providing services has caused the sudden increase in the amount of the services that are operationally similar. The dissimilarity in such services are observed based on the characteristics standards of their service quality. Consequently a thought-provoking issue in the perspective of the service-configuration is based on the choosing the accurate client services according to the user demands for which the acquired compound services guarantees the noncomputational demands on the ground of comprehensive service quality constraints. The procedure carried out in the paper presents the novel algorithm for the service composition/configuration for enhancing the quality of service of the compound /composite computing services. The algorithm focusses in improve the service configuration feasibility and optimality and minimize the configuration time. The algorithm is totally based on the limits in the configuration strategies that relies on the Pareto-Dominance and the quality of service decomposition (PD-QOSD). The quantity of the service arrangements is expected to increase according to the service count and quality of service characteristics in case of PD and whereas the QOSD takes care of the feasibility of the arrangements during the high restrictions imposed by users on the constraints of the comprehensive quality of service. The validation outcomes prove that the novel algorithm developed function efficiently on the basis of the time required for composition, the optimality, as well as the search space.
\end{abstract}

Keywords: Compound Services, Composition of Services, QOSD, PD, QOS Enhancements

\section{Introduction}

Providing service according to the requirements of the user is basically taken care computing fixed to facilitate the service provision and are termed as the service computing, the fundamental and basic elements involved in this type of computing are the basic devices for computing, and the software elements dispersed in the web. These process of service provisioning are completely made possible by the procedure that are available to arrange the service provisions, nowadays the process has become more tedious and perplexing in the perspective of the service-configuration. According to the author Papazoglou, Mike P., et al [1] the service computing is defined as the "A software entity that provides a set of functionalities defined in a 
Journal of ISMAC (2020)

Vol.02/ No. 02

Pages: 118- 128

http://irojournals.com/iroismac/

DOI: https://doi.org/10.36548/jismac.2020.2.005

service description called interface that includes both the functional and the non-functional properties of the service" the functional properties sorts out the optimal clients services that satisfies the demands of the user. Though the operationally similar these services differ in the QOS characteristics and the nonfunctional chooses the best operationally alike services to satisfy the user needs.

The environments of the service computing are categorized as concrete and abstract where the former denotes the service that are executable the latter denotes the set of service that are concrete with similar capabilities. These services possess same parameters for the input and the output and different quality of service characteristics. The very fundamental service are combined to form novel services called composite or the compound services. This is basically done to improve the capabilities of the services as in Khanouche, et al [2]. The author Liu, et al [3] presented the swift progress in the computing used in the services, IoT, Cloud Computing, and BDA has caused a considerable increase in the quantity of the functionality similar services that differ in its QOS characteristics. Most challenging issue as stated in the Zheng et al [4] is based on method of selecting the optimal client services that are essential in guaranteeing the procedure followed in the arranging the services. The choice indeed is based on the multiple of computable characteristics that enables to assess the complete quality of the service rendered to the users. Certainly the characteristics of the QOS that commonly used in the previous researches to describe the QOS in the application level are availability, reliability, cost, response time, and reputation. The issue of the composition of services consists in particular of finding the utmost appropriate services according to user demand in such a way that the subsequent combined service fulfills, both the functional and the nonfunctional demands that are imposed in form of comprehensive constraints over the service quality of the arrangements done for the service provisions.

The constraints or the restrictions may be either the average cost that should not cross the limit or the reliability level set, to satisfy the users. The paper scopes to developing an algorithm that provides a better service composition such that the QOS is guaranteed. The algorithm mainly focusses on the perspective of the service computing to minimize the composition search space to minimize the execution time and heighten the optimality.

The PD is applied in the proposed algorithm to minimize the search space of the arrangement. It filters out the controlled compound /composite services according to the values of the QOS as in the Alrifai, et al [5] that presents the steps in making the choices on the services of skyline for the service configurations in the web to guarantee the QOS, Chen, et al [6] who presented the "A partial selection methodology for efficient QoS-aware service composition." Wang, et al [7] that performed the procedures to provide an ensured QOS in arranging the services for the "cyber physical social system" Yu, et al [8] that provides the method of the choosing the client service with effective skyline service processing. 
Journal of ISMAC (2020)

Vol.02/ No. 02

Pages: $118-128$

http://irojournals.com/iroismac/

DOI: https://doi.org/10.36548/jismac.2020.2.005

Nevertheless the amount of these combined, services exponentially increases with the amount of service arranged. In addition, in the case of the global QoS constraints of highly restrictive users, the QOSD service composition methods may result in harsh local QoS constraints resulting in a reduction in the feasibility of composition as in the Cho et al [9], who performed the Adaptive service selection according to the service density in multiple QoS aspects." Hwang et al [10] that conducted the. "Service selection for web services with probabilistic QoS." Liu, et al [11] that has performed the "Reliable Web service composition based on QoS dynamic prediction" and the Yuan et al [12] that has performed "Dynamic Service Selection Based on Adaptive Global QoS Constraints Decomposition."

Further the Raj, Jennifer S. et al [13] performed the QOS attained path to route the data sensed by the wireless sensor that are employed as the basic elements in the IOT for providing service for the smart environments. Bhalaji, N. et al [14] devised the defense mechanism for the flying- wireless network employing the block chain service. The security based on the block chain allowed the user to have an expected quality of service. Kumar, T. Senthil et al [15] performed the resource allocation process using the nmetaheuristics to improve the quality of service of the IoT that is integrated with the FOG. Haoxiang et al [16] utilized the soft computing techniques to develop a quality of service enhanced protocol o identify the path to route the service required by the vehicular networks.

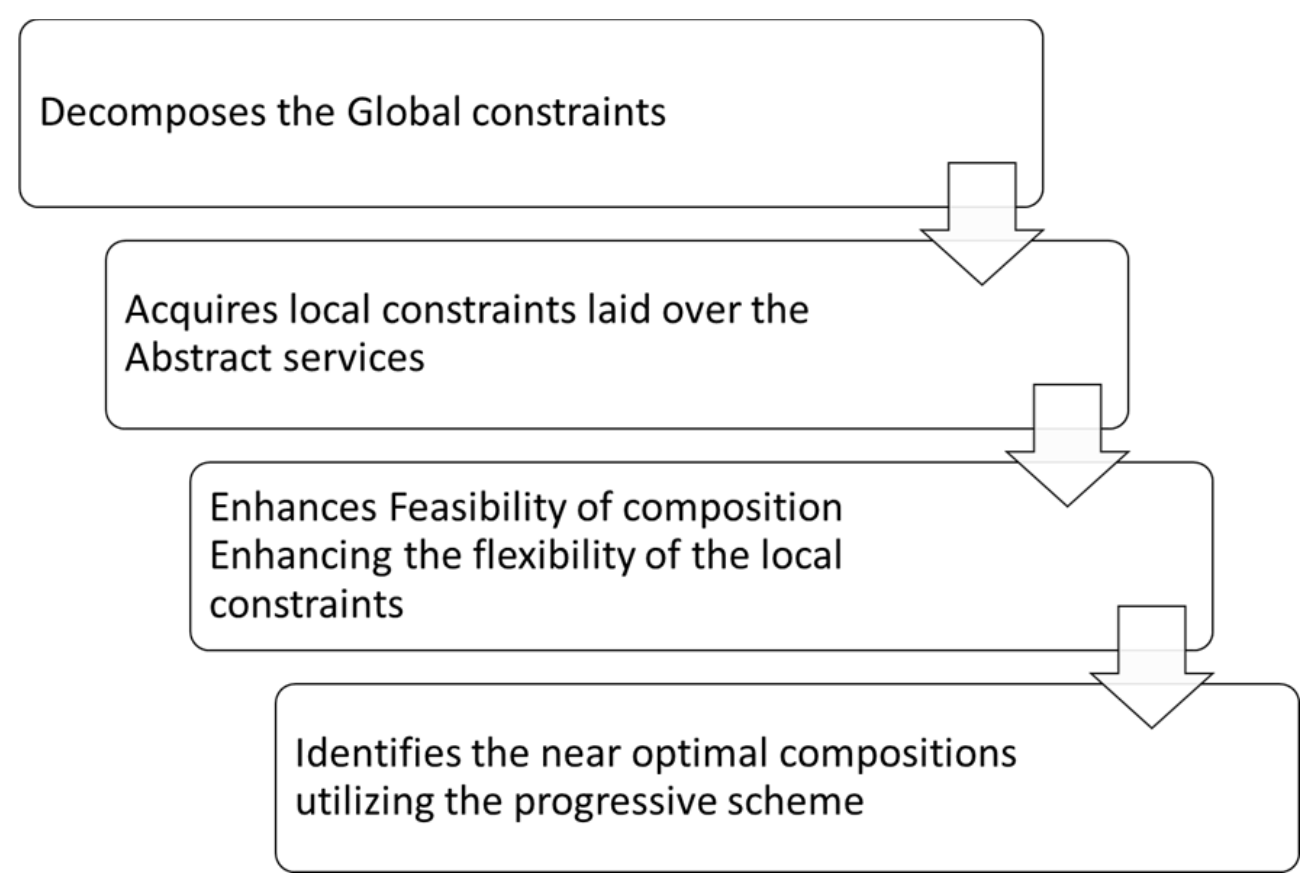

Figure .1 Flow of Proposed Algorithm

ISSN: 2582-1369 (online) 
Journal of ISMAC (2020)

Vol.02/ No. 02

Pages: $118-128$

http://irojournals.com/iroismac/

DOI: https://doi.org/10.36548/jismac.2020.2.005

To overcome the limitations, the method that minimizes the value of the local QOS limitation is followed, to thereby enhance the feasibility in arrangement. The novel algorithm framed in the paper, decomposes the constraints that are global and acquire the constraints that are local and carried out on the configuration of the abstract services and increase the feasibility in the next stage by increasing the feasibility of the local constraint and minimizing the amount of the client services. This decreases the time taken for composition and heightens the optimality following the above two steps a closer optimal configuration for service is identified on the basis of quality of service utilizing the progressive scheme. The figure. 1 shows the stages of the proposed work.

The rest of the paper is arranged with the novel algorithm for providing the QOS enhanced Service compositions in two, the performance analysis in three and the conclusion in four.

\section{Proposed Work}

The process of composing the services includes the group of abstract services that are linked to the well planned service arrangements. This is further made concrete by selecting the client services for every service that is abstract and makes sure the QOS is guaranteed according to the user needs with an increased value of utility. All probable arrangements of the client's services are examined to identify the optimal service with improved QOS. The approaches performed so for consumes more time for configuring a service due to the exponential increase experienced in its search space, so the paper proposes the algorithm that configures the services within a rational time along with a assured QOS.As depicted in the figure.1 the proposed algorithm starts with the decomposition of the QOS constraints that are global. The global constraints for the QOS denoting the non-functional necessities of the users are disintegrated into the local constraints and every local constraint is allocated to the attribute of the quality of service. The algorithm below in figure. 2 depicts the process of decomposition followed.

ISSN: 2582-1369 (online) 
Journal of ISMAC (2020)

Vol.02/ No. 02

Pages: $118-128$

http://irojournals.com/iroismac/

DOI: https://doi.org/10.36548/jismac.2020.2.005

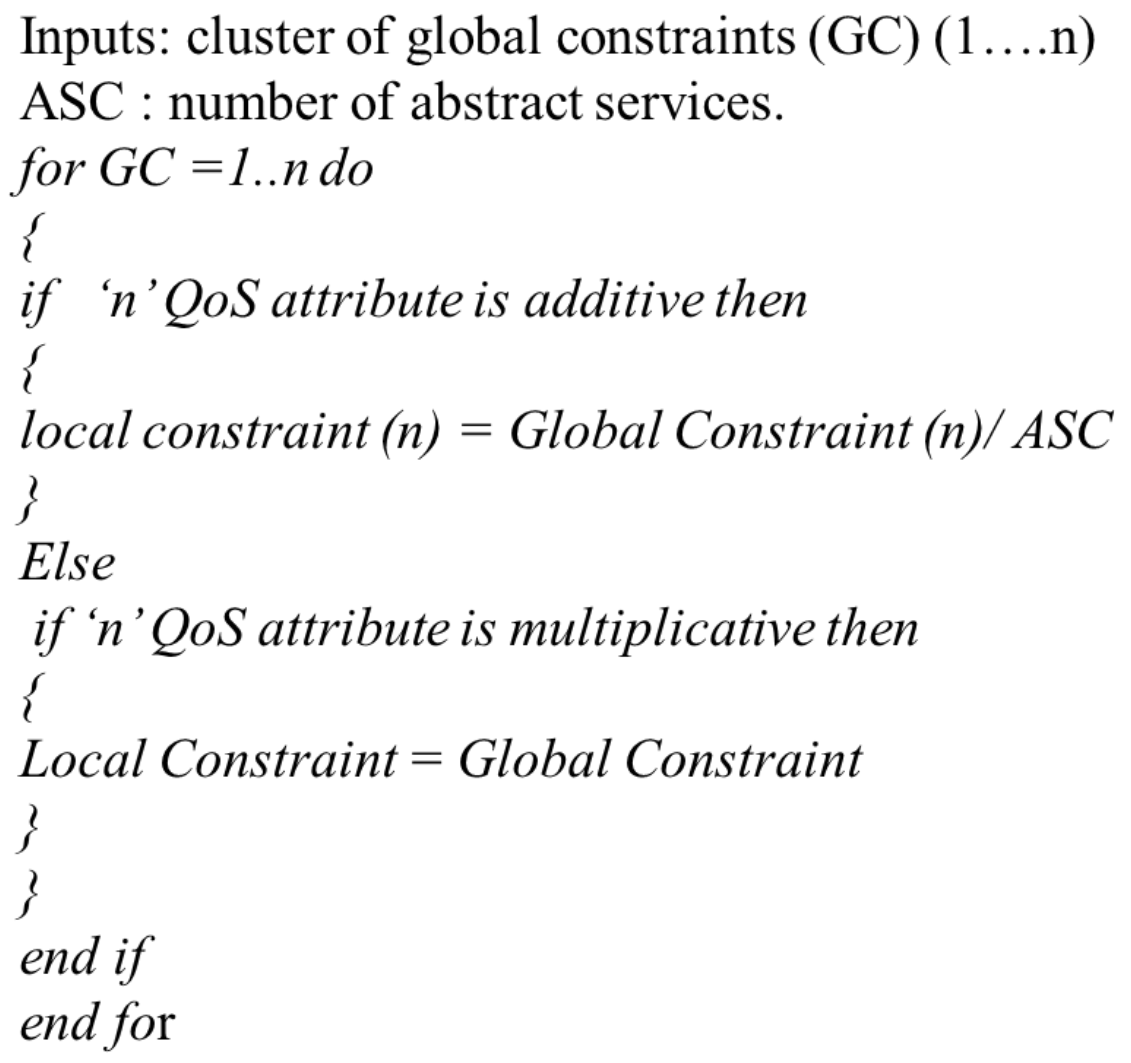

Outputs: Group of Local Constraints (1...i)

Figure.2Decomposition Process

The next stage filters the services including the procedures of choosing the services according to the flexibility of the quality of service and identifying the set of optimal Pareto composition of service is carried out. The algorithm. 1 below in the figure .3 describes the choosing process.

ISSN: 2582-1369 (online) 
Journal of ISMAC (2020)

Vol.02/ No. 02

Pages: 118- 128

http://irojournals.com/iroismac/

DOI: https://doi.org/10.36548/jismac.2020.2.005

\author{
Input : Local Constraint \\ LC(1...i) \\ Flexible Factors $\{\mathrm{FF}(1 \ldots . . \mathrm{i})\}$ \\ For $L C=1 \ldots i$ \\ Do \\ \{ \\ $L C_{F F}=L C x F F$ \\ if ' $i$ ' QoS attribute is -ve then \\ $L C_{F F}($ threshold $)=L C+L C_{F F}$ \\ Else \\ $L C_{F F}($ threshold $)=L C-L C_{F F}$ \\ \} \\ End if \\ End for \\ Output $L C_{F F}$ (threshold)
}

Figure.3 Selection Process

The selection process is followed by the identification process that utilizes the progressive composition scheme, the strategy sorts out the initial partial set and integrated with the optimal Pareto services, this is done in order to identify the next partial set the steps are iterated to identify all probable set. The above process is performed using the partial-composition procedure. The figure. 4 below shows the P-CP for the identifying the optimal Pareto sets.

ISSN: 2582-1369 (online) 
Journal of ISMAC (2020)

Vol.02/ No. 02

Pages: 118- 128

http://irojournals.com/iroismac/

DOI: https://doi.org/10.36548/jismac.2020.2.005

$$
\begin{aligned}
& \text { Inputs: OPCS sequenced based on } \\
& \text { utility value of their concrete } \\
& \text { services. } \\
& \text { OPC = null ; IN = initial node } \\
& \text { Node extended }(\mathrm{NE})=\{\mathrm{IN}\} \\
& \text { initialize the Parent; } \\
& \text { while } N E=\text { null }
\end{aligned}
$$

Do

Terminate IN from NE

if IN not dominated

Add IN OPCS

end if

for every child node (CN of IN)

Do

$$
\begin{aligned}
& P T(C N)=P T(C N)-1 \\
& \text { if } P T(C N)==0 \text { then } \\
& \text { Add } C N \text { to } N E \\
& \text { end if } \\
& \text { end for } \\
& \text { end while }
\end{aligned}
$$

\section{Outputs: set of OPC}

Figure.4 P-CP pseudo code for Identifying the Optimal Pareto CS

The P-CP uses the structure of the extended points as buffer to reserve the nodes that are extended into the expansion lattice. This structure of the nodes extended decides the sequence of evaluation for the nodal points that do not have a parent or a child relationship. The nodes assigned as parents denotes the services that are concrete index number that does not match the index of the concrete service assigned to the root node. The list holding the concrete services that are under the guide ship of the OPCS is arranged in the downward sequence, based on the value of utility. This kind of arranging improves its performance and as well as allows to have an optimal configuration of services and in turn minimizes the amount of the services that affects the number of comparisons performed while exploring nodes in the lattice expanded.

ISSN: 2582-1369 (online) 
Journal of ISMAC (2020)

Vol.02/ No. 02

Pages: $118-128$

http://irojournals.com/iroismac/

DOI: https://doi.org/10.36548/jismac.2020.2.005

\section{Performance Evaluation}

The novel algorithm based on the PD-QOSCD mechanism used to enhance the QOS constraints is evaluated using JAVA language and executed in the PC with the Ryzen $25002^{\text {nd }}$ generation, windows 10, with the frequency of $2.5 \mathrm{GHz}$ and RAM of 4GB, The real time data (QWS) are used for simulating the dataset has 19 attributes for QOS an 2500 services that are concrete.

The validation results depicting the performance is compared with the other algorithm such as the BSC [8] and PBPSS [6] on the basis of the time taken in configuration, utility value search space the PD, engaged enables to minimize the search space for the configuration.

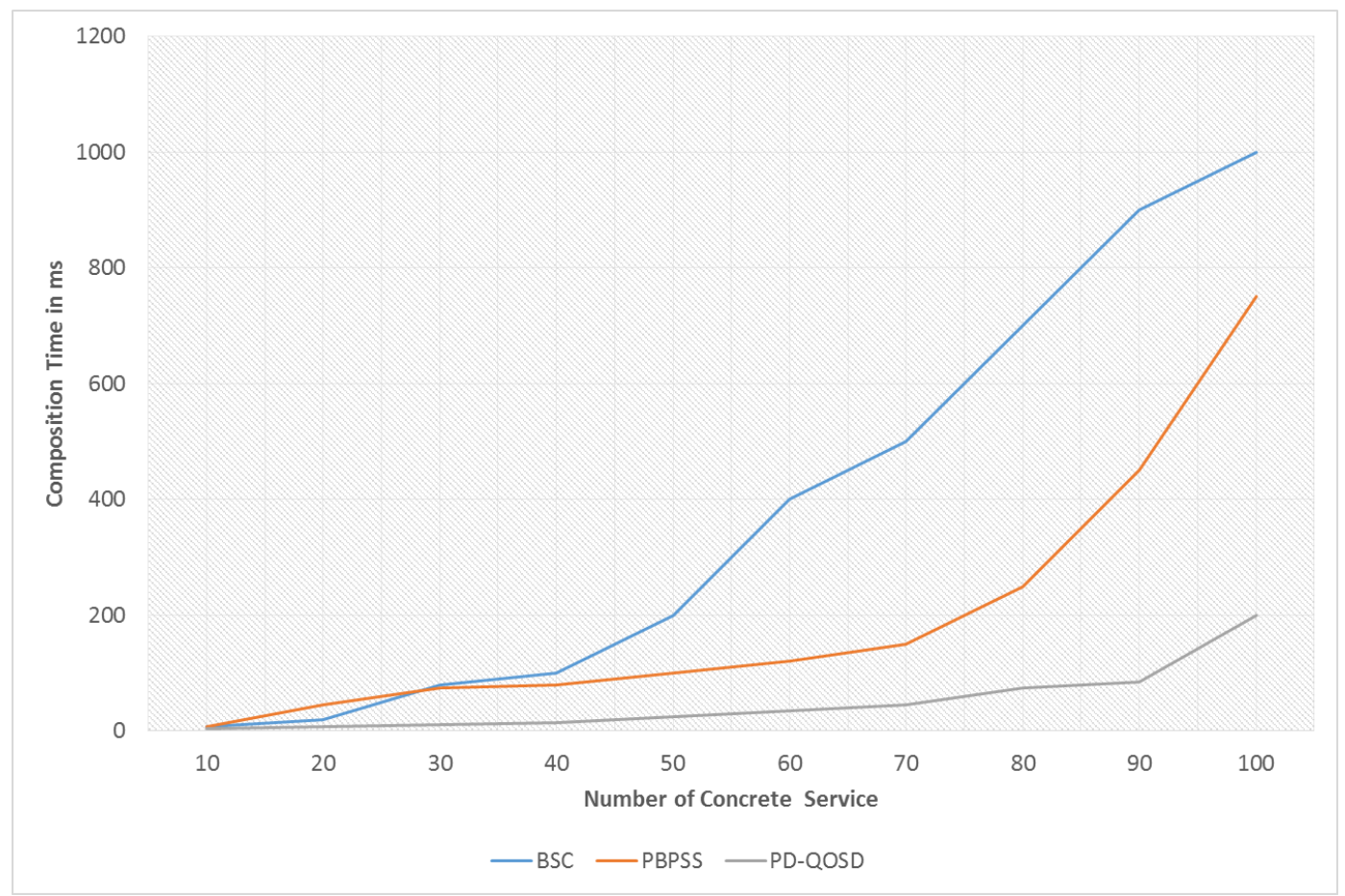

Figure.5 Time for Composition

The figure. 5 is the composition time achieved by the PD-QOSD and the other two existing algorithms for the amount of concrete services, varying from 10 to 100 , the results shows that the proposed novel algorithm has much reduced and reasonable composition time compared to the other two.

ISSN: 2582-1369 (online) 
Journal of ISMAC (2020)

Vol.02/ No. 02

Pages: 118- 128

http://irojournals.com/iroismac/

DOI: https://doi.org/10.36548/jismac.2020.2.005

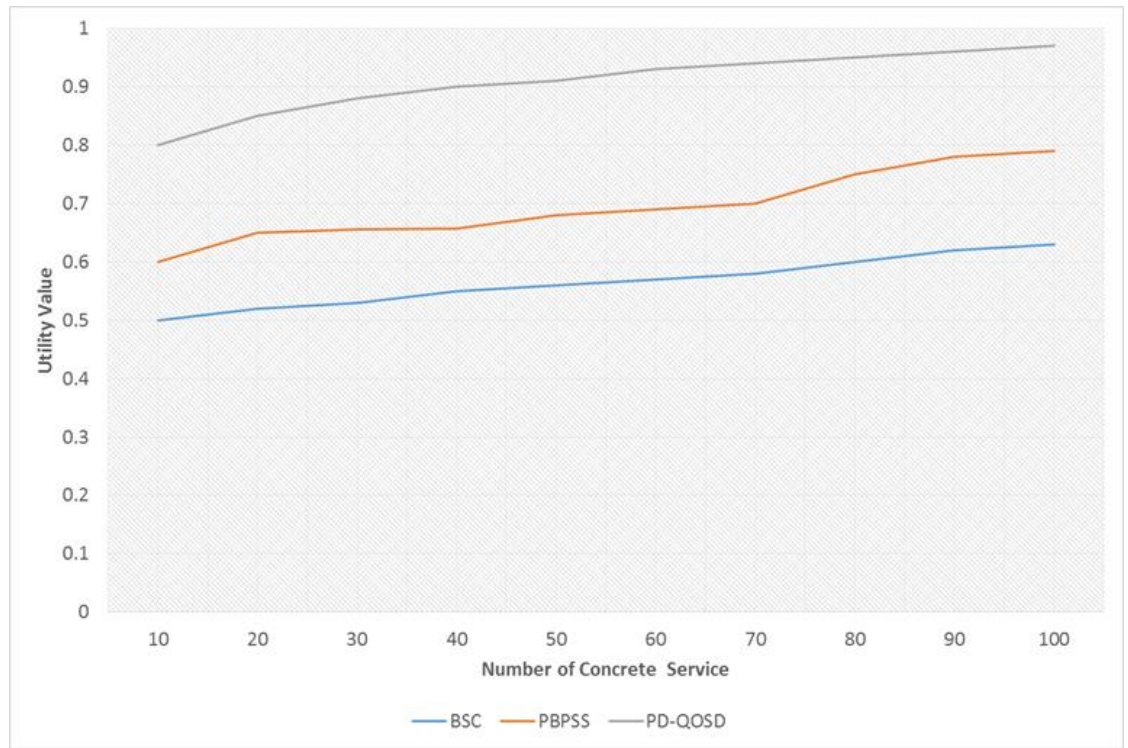

Figure.6 Utility Value

The utility value observed for all the three algorithm for the composition of services is observed in figure. 6 and comparison shows that the proposed method is very good utility value compared to the other application of the other algorithm.

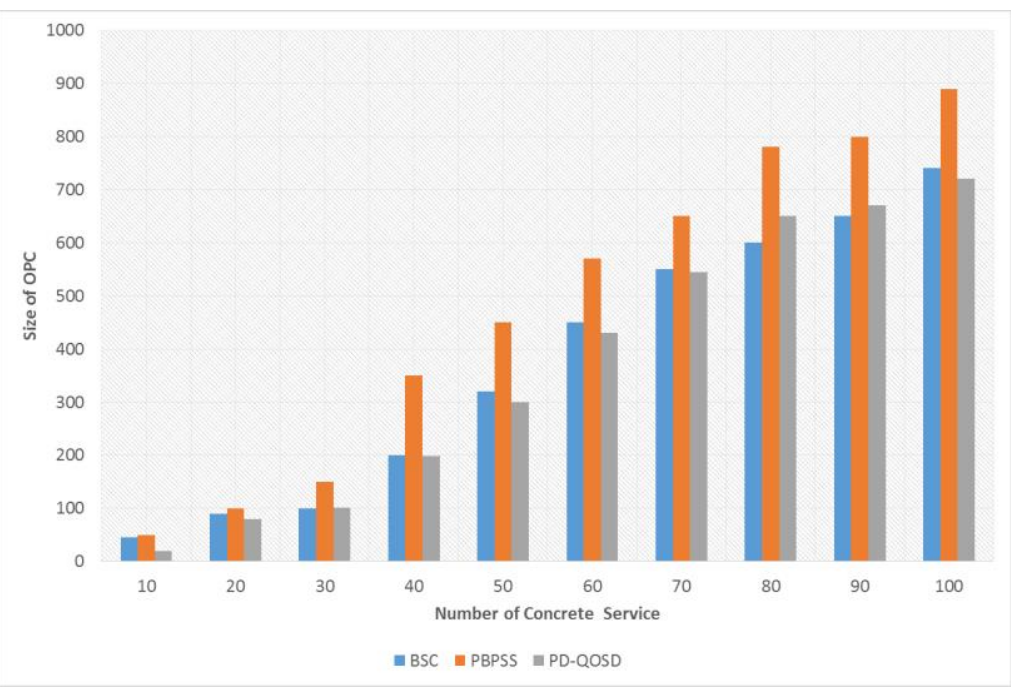

Figure.7 OPC size 
Journal of ISMAC (2020)

Vol.02/ No. 02

Pages: 118- 128

http://irojournals.com/iroismac/

DOI: https://doi.org/10.36548/jismac.2020.2.005

The size of OPC varying number of concrete services are observed in figure.7 and compared with the other two exiting algorithms, the number of OPC enhances with the number of the attributes of QOS. The results observed for the proposed were better compared to the other two.

\section{Conclusion}

The novel algorithm proposed in the paper enables to achieve an enhanced quality of service by minimize the time taken for the composition of the services and search space and enhancing the utility value to maintain the optimality. The evaluation results observed proves the performance of the proposed algorithm over the other two existing methods such as the BCS, PBPSS. In future the paper is carries out the procedure to handle the issues related to the value variations that occurs in the QOS along with the planning of the composition, as the paper in the present method assumes that the composition plan is well planned.

\section{References}

[1] Papazoglou, Mike P., and Willem-Jan Van Den Heuvel. "Service oriented architectures: approaches, technologies and research issues." The VLDB journal 16, no. 3 (2007): 389-415.

[2] Khanouche, Mohamed Essaid, Ferhat Attal, Yacine Amirat, Abdelghani Chibani, and Moussa Kerkar. "Clustering-based and QoS-aware services composition algorithm for ambient intelligence." Information Sciences 482 (2019): 419-439.

[3] Liu, Zhi-Zhong, Dian-Hui Chu, Zong-Pu Jia, Ji-Quan Shen, and Lei Wang. "Two-stage approach for reliable dynamic Web service composition." Knowledge-Based Systems 97 (2016): 123-143.

[4] Zheng, Zibin, Yilei Zhang, and Michael R. Lyu. "Investigating QoS of real-world web services." IEEE transactions on services computing 7, no. 1 (2012): 32-39.

[5] Alrifai, Mohammad, Dimitrios Skoutas, and Thomas Risse. "Selecting skyline services for QoS-based web service composition." In Proceedings of the 19th international conference on World wide web, pp. 11-20. 2010.

[6] Chen, Ying, Jiwei Huang, Chuang Lin, and Jie Hu. "A partial selection methodology for efficient QoS-aware service composition." IEEE Transactions on Services Computing 8, no. 3 (2014): 384-397.

[7] Wang, Shangguang, Ao Zhou, Mingzhe Yang, Lei Sun, and Ching-Hsien Hsu. "Service composition in cyber-physical-social systems." IEEE Transactions on Emerging Topics in Computing (2017).

[8] Yu, Qi, and Athman Bouguettaya. "Efficient service skyline computation for composite service selection." IEEE Transactions on Knowledge and Data Engineering 25, no. 4 (2011): 776-789. 
Journal of ISMAC (2020)

Vol.02/ No. 02

Pages: $118-128$

http://irojournals.com/iroismac/

DOI: https://doi.org/10.36548/jismac.2020.2.005

[9] Cho, Jae-Hyun, Han-Gyu Ko, and In-Young Ko. "Adaptive service selection according to the service density in multiple QoS aspects." IEEE Transactions on Services Computing 9, no. 6 (2015): 883-894.

[10] Hwang, San-Yih, Chien-Ching Hsu, and Chien-Hsiang Lee. "Service selection for web services with probabilistic QoS." IEEE transactions on services computing 8, no. 3 (2014): 467-480.

[11] Liu, Zhi Zhong, Zong Pu Jia, Xiao Xue, and Ji Yu An. "Reliable Web service composition based on QoS dynamic prediction." Soft Computing 19, no. 5 (2015): 1409-1425.

[12] Yuan, Yuan, Weishi Zhang, Xiuguo Zhang, and Huawei Zhai. "Dynamic Service Selection Based on Adaptive Global QoS Constraints Decomposition." Symmetry 11, no. 3 (2019): 403.

[13] Raj, Jennifer S. "QoS optimization of energy efficient routing in IoT wireless sensor networks." Journal of ISMAC 1, no. 01 (2019): 12-23.

[14] Bhalaji, N. "Qos and Defense Enhancement Using Block Chain for Fly Wireless Networks." Journal of trends in Computer Science and Smart technology (TCSST) 1, no. 01 (2019): 1-13.

[15] Kumar, T. Senthil. "Efficient resource allocation and QOS enhancements of IoT with FOG network." J ISMAC 1 (2019): 101-110.

[16] Haoxiang, Wang, and S. Smys. "QOS Enhanced Routing Protocols For Vehicular Network Using Soft Computing Technique." Journal of Soft Computing Paradigm (JSCP) 1, no. 02 (2019): 91-102.

\section{Authors Biography}

The author Dr. A. Pasumponpandian, Professor, has completed his UG and PG degree from the reputed colleges and works as Professor, in Computer Science Engineering, iKGiSL Institute of Technology, Coimbatore, India. His areas of research includes Social Networks, Wireless Networks, Internet of Things, Computer Networks, Mobile Communication, Robotics and Electrical Infrastructure, Mobile APIs, Data Analysis and Visualization.

ISSN: 2582-1369 (online) 